\title{
Recommendations for radiosurgery and stereotactic ablative radiotherapy terminology to guide clinical and research practices for the medical oncologist, radiation oncologist, and surgeon
}

\author{
Mohamed H. Khattab ${ }^{1,2} \cdot$ Alexander D. Sherry ${ }^{3} \cdot$ Michael L. Freeman $^{1} \cdot$ Guozhen Luo ${ }^{1} \cdot$ Evan C. Osmundson ${ }^{1}$. \\ Albert Attia ${ }^{1}$. Anthony J. Cmelak ${ }^{1}$
}

Received: 12 March 2020 / Accepted: 3 May 2020 / Published online: 15 May 2020

(C) Springer-Verlag GmbH Germany, part of Springer Nature 2020

Stereotactic radiosurgery (SRS), stereotactic ablative radiotherapy (SABR), and stereotactic body radiation therapy (SBRT) are tools used independently or in conjunction with systemic therapies and have transformed oncologic practice. With the emergence of these therapeutic modalities, and in part due to a lack of established guidelines differentiating these treatments, the term "radiosurgery" is frequently erroneously ascribed to procedures marketed to the medical community and patients as having ablative radiobiologic effects. This may not always be the case and depends on dose, fractionation, and tumor type. At times, such marketing appears arbitrary and unscientific. To the dismay of the radiation oncology community, utilization of stereotactic targeting has been frequently misconstrued as delivery of ablative dosing. Prior attempts have been made to focus nomenclature, notably such as those of Loo and colleagues who argued that the term SABR more precisely describes the treatment modality than SBRT [1]. Since that time and with continued advances in technology, further terminology ambiguities have arisen including (hypo)fractionated stereotactic radiosurgery, multisession radiosurgery, and (hypo)fractionated stereotactic radiotherapy, all of which are frequently used interchangeably with SABR and SBRT. The ramifications of inconsistencies in nomenclature are vast and have implications to standard

Mohamed H. Khattab

mohamed.khattab@vumc.org

1 Department of Radiation Oncology, Vanderbilt University Medical Center, 2220 Pierce Avenue, Preston Research Building, Rm B-1003, Nashville, TN 37232-5671, USA

2 Vanderbilt-Ingram Cancer Center, 2220 Pierce Avenue, Preston Research Building, Rm B-1003, Nashville, TN 37232-5671, USA

3 Vanderbilt University School of Medicine, Nashville, TN 37232-5671, USA clinical practice, interpretation of previously reported outcomes comparing radiosurgery to competing modalities, and for those of future trial design. In addition, billing and compensation for these procedures are altered by the application of these nomenclatures, and it is unknown whether compensation and/or marketing drives the use of the term radiosurgery when treatment is non-ablative. For example, fractionated radiosurgery according to US insurance companies is typically reimbursed only as radiosurgery if the number of fractions is no greater than 5; by contrast, some countries in Europe have a fraction limitation of 8 , and regimens of 8 fractions may be equivalently ablative.

In 2007, the neurosurgery community independently developed nomenclature guidelines in an attempt to address this issue, though these were significantly limited and not inclusive of the indications for stereotactic targeting used by radiation oncologists and thus have not been uniformly followed [2]. Additionally, recent improvements and increased utilization of linear accelerator-based stereotactic radiation and the ability to fractionate delivery using new Gamma Knife technologies, as well as an emerging understanding of how ablative dosing may allow for priming of an endogenous systemic response, justify an impetus for new guidelines, which remain conspicuously absent from the radiation oncology community.

There are a number of consequences from the lack of uniform terminology. For example, a controversial national cancer database study comparing radiofrequency ablation (RFA) versus radiation for localized hepatocellular carcinoma reported superiority of radiofrequency ablation to SBRT in the Journal of Clinical Oncology, based on questionable labeling of the radiotherapy delivered to which RFA was compared [3]. Although the study authors described the radiation arm as SBRT, $36 \%$ of patients in the radiation cohort were treated with biologically effective doses (BED) consistent with palliative, non-SBRT regimens, invalidating the study's primary conclusions and generating significant confusion [4]. More recently, 
data from the phase III NRG Oncology/RTOG 0631 trial comparing 16-18 Gy in 1 fraction versus 8 Gy in 1 fraction for treatment of spine metastases were presented at the 2019 ASTRO annual meeting [5]. Three-month pain control was not found to be superior in the higher dose arm, prematurely suggesting that conventional radiation is more appropriate for palliation than "radiosurgery." However, spine stereotactic radiosurgery is usually prescribed between 18 and 26 Gy when delivered by a single fraction, raising a question as to whether this study truly compared radiosurgery versus conventional radiotherapy or two conventional radiotherapy regimens [6]. An ongoing phase III clinical trial led by Sahgal and colleagues at Sunnybrook aims to delineate possible benefits in SBRT dosing (NCT02512965) using cohorts with different BEDs, though there is also a question whether their SBRT cohort has accomplished the necessary BED to be truly ablative.

The ramification of these inconsistencies is significant as they obfuscate the biological mechanisms that differentiate radiosurgery from other stereotactic-guided radiation delivered in one to five fractions. Unlike traditional radiotherapy, radiosurgery is hypothesized to more strongly activate ceramideinduced apoptosis. This mechanism may be more cytotoxic in tissues, such as endothelia, with significant intracellular concentrations of acid sphingomyelinase, the key enzyme in the ceramide apoptosis pathway [7]. Tumors dependent on endothelial cell survival, such as renal cell carcinoma, may therefore be radiosensitive at radiosurgical dosing, but radioresistant when treated with conventional radiotherapy [8]. Furthermore, in the era of immunotherapy, where the production of novel epitopes is thought to be the primer for an endogenous immune response, this distinction is particularly paramount, as studies of "SBRT" utilizing non-radiosurgical regimens may mislead the oncology community as well as payers.

In the context of these important clinical and biological studies, a number of outstanding questions remain (Table 1).

First, if the RTOG 0631 regimen were considered radiosurgical, would this regimen best be described as SRS/

Table 1 List of primary outstanding questions in radiosurgery and ablative radiotherapy

Question

1. What defines and distinguishes SRS, SBRT, SABR, and SSRT?

2. What should guide the determination of hypofractionated stereotactic radiotherapy versus radiosurgery?

3. How should the terms multifraction versus multisession be distinguished?

4. Should there be a fraction limited (i.e., 5 fractions) for radiosurgery?

5. What factors should be incorporated into the determination of ablative radiation?

6. How should the limitations of BED be addressed in comparing radiosurgical regimens?
SBRT, as the authors write, or SABR or spine stereotactic radiosurgery (SSRS)? How should these terms be differentiated, if at all?

Second, how should the current interchangeable use of various terms including SRS, multisession SRS, multifraction SRS, (hypo)fractionated SRS, SSRS, and (hypo)fractionated stereotactic radiotherapy be addressed? What is the appropriate context for each of these seemingly distinct terms? What criteria should be employed to determine this nomenclature? Dose, stereotactic targeting, and steepness of dose gradient with appropriate R-50 conformity (the volume covered by $50 \%$ of the prescription divided by the planned target volume given that the prescription dose covers at least $95 \%$ of the planned target volume) should likely be considered. Furthermore, Dmax/hotspot greater than $120 \%$ appears worthy of inclusion as well $[9,10]$. Should the assignment of this terminology also account for histology, relative radiosensitivity, and BED? To expand upon this concept, the treatment of vestibular schwannoma with 25 Gy in 5 fractions has a BED of $67 \mathrm{~Gy}$, similar to the $65 \mathrm{~Gy}$ BED of the standard radiosurgical dosing 12.5 Gy in 1 fraction, assuming an alpha/beta ratio of 3 . Meanwhile, the treatment of functional pituitary adenoma, using the same 25 Gy in 5 fraction regimen with a BED of $67 \mathrm{~Gy}$, appears significantly less than the 126 Gy BED of standard of care radiosurgery using 18 Gy in 1 fraction $[11,12]$. Therefore, should the 25 Gy in 5 fraction regimens for vestibular schwannoma be termed multifraction SRS or multisession SRS, and the 25 Gy in 5 fraction regimen for functional pituitary adenoma be called hypofractionated stereotactic radiotherapy? Or, should the 25 Gy in 5 fraction regimens for pituitary adenoma be described as multisession radiosurgery, as per Adler et al. [13]?

Complicating the matter further is how to determine whether a fractionated regimen is equivalent to single fraction SRS. One common notion is that a fractionated regimen with BED exceeding the BED of a single fraction treatment is radiosurgical. Some groups contend that BED exceeding $100 \mathrm{~Gy}$ is considered biologically radiosurgical, such as for lung cancer, although different histology may have different thresholds and not all single fraction SRS regimens have a BED greater than 100 Gy $[14,15]$. Therefore, should these criteria include a site-specific requirement for a minimum BED or BED exceeding that of the standard of care single fraction SRS course? Although linear quadratic modeling calculates BED for the comparison of regimens, the assumptions of this model may not apply at radiosurgical doses, and thus incorporating what appears to be a critical component of defining radiosurgery may introduce significant radiobiological flaws and remains hotly debated, as well as alternatives such as the universal survival curve [16-18].

Thus, there is a need to standardize what dosing regimens should be considered radiosurgery versus radiotherapy guided by stereotactic targeting, given the divergent biological 
mechanisms and despite delivery in hypofractionated or ultrahypofractionated schedules. In the era of linear accelerator-based SRS, SABR, and immunotherapy, we call for the development of a standardized nomenclature of radiosurgery by the radiation oncology and neurosurgery community in order to mitigate current obstacles to understanding the current radiosurgical literature, development of future radiosurgical clinical trials, and guiding clinical practice for medical oncologists, radiation oncologists, and oncologic surgeons. Further investigation and interdisciplinary consensusbuilding of a proposed framework is desperately needed.

\section{Compliance with ethical standards}

Conflicts of interest Mohamed Khattab has received research grants from Varian Medical Systems and Brainlab, Inc. Alexander Sherry declares that he has no conflict of interest. Michael Freeman declares that he has no conflict of interest. Guozhen Luo declares that she has no conflict of interest. Evan Osmundson declares that he has no conflict of interest. Albert Attia has received research funding from Brainlab, AstraZeneca, and Novocure. Anthony Cmelak declares that he has no conflict of interest.

Ethical approval This is an Editorial Comment that does not include animal or human data meriting institutional review board review.

Informed consent No human subjects were a component of this Editorial Comment.

\section{References}

1. Loo B, Chang J, Dawson L et al (2011) Stereotactic ablative radiotherapy: what's in a name? Pract Radiat Oncol 1:38-39. https://doi. org/10.1016/j.prro.2010.07.001

2. Barnett GH, Linskey ME, Adler JR, Cozzens JW, Friedman WA, Heilbrun MP, Lunsford LD, Schulder M, Sloan AE, American Association of Neurological Surgeons, Congress of Neurological Surgeons Washington Committee Stereotactic Radiosurgery Task Force (2007) Stereotactic radiosurgery - an organized neurosurgery-sanctioned definition. J Neurosurg 106:1-5. https:// doi.org/10.3171/jns.2007.106.1.1

3. Rajyaguru DJ, Borgert AJ, Smith AL, Thomes RM, Conway PD, Halfdanarson TR, Truty MJ, Kurup AN, Go RS (2018) Radiofrequency ablation versus stereotactic body radiotherapy for localized hepatocellular carcinoma in nonsurgically managed patients: analysis of the national cancer database. J Clin Oncol 36: 600-608. https://doi.org/10.1200/JCO.2017.75.3228

4. Shinde A, Jones BL, Chen YJ, Amini A (2018) Radiofrequency ablation versus stereotactic body radiotherapy for localized hepatocellular carcinoma: does radiation dose make a difference? J Clin Oncol 36:2566-2567. https://doi.org/10.1200/JCO.2018.78.6012

5. Ryu S, Deshmukh S, Timmerman R et al (2019) Radiosurgery compared to external beam radiotherapy for localized spine metastasis: phase III results of NRG oncology/RTOG 0631. Int J Radiat Oncol Biol Phys 105:S2-S3
6. Robin AM, Yamada Y, McLaughlin LA et al (2017) Stereotactic radiosurgery: the revolutionary advance in the treatment of spine metastases. Clin Neurosurg 64:59-65. https://doi.org/10.1093/ neuros/nyx 222

7. Kolesnick R, Fuks Z (2003) Radiation and ceramide-induced apoptosis. Oncogene 22:5897-5906. https://doi.org/10.1038/sj.onc. 1206702

8. De Meerleer G, Khoo V, Escudier B et al (2014) Radiotherapy for renal-cell carcinoma. Lancet Oncol 15:e170-e177. https://doi.org/ 10.1016/S1470-2045(13)70569-2

9. Zeng ZC, Seong J, Yoon SM, Cheng JCH, Lam KO, Lee AS, Law A, Zhang JY, Hu Y (2017) Consensus on stereotactic body radiation therapy for small-sized hepatocellular carcinoma at the 7th AsiaPacific Primary Liver Cancer Expert Meeting. Liver Cancer 6:264 274. https://doi.org/10.1159/000475768

10. Sanford L, Molloy J, Kumar S, Randall M, McGarry R, Pokhrel D (2019) Evaluation of plan quality and treatment efficiency for single-isocenter/two-lesion lung stereotactic body radiation therapy. J Appl Clin Med Phys 20:118-127. https://doi.org/10.1002/acm2. 12500

11. Khattab M, Sherry A, Whitaker R et al (2019) A retrospective cohort study of longitudinal audiologic assessment in single and fractionated stereotactic radiosurgery for vestibular schwannoma. Neurosurgery 85:E1078-E1083. https://doi.org/10.1093/neuros/ nyz219

12. Sherry A, Khattab M, Xu M et al (2019) Outcomes of stereotactic radiosurgery and hypofractionated stereotactic radiotherapy for refractory Cushing's disease. Pituitary 22:607-613. https://doi.org/ 10.1007/s11102-019-00992-6

13. Adler JR, Gibbs IC, Puataweepong P, Chang SD (2006) Visual field preservation after multisession cyberknife radiosurgery for perioptic lesions. Neurosurgery 59:2442-2454. https://doi.org/10. 1227/01.NEU.0000223512.09115.3E

14. Robinson CG, Dewees TA, El Naqa IM et al (2013) Patterns of failure after stereotactic body radiation therapy or lobar resection for clinical stage i non-small-cell lung cancer. J Thorac Oncol 8: 192-201. https://doi.org/10.1097/JTO.0b013e31827ce361

15. Olsen JR, Robinson CG, El Naqa I et al (2011) Dose-response for stereotactic body radiotherapy in early-stage non-small-cell lung cancer. Int J Radiat Oncol Biol Phys 81:e299-e303. https://doi. org/10.1016/j.ijrobp.2011.01.038

16. Park C, Papiez L, Zhang S, Story M, Timmerman RD (2008) Universal survival curve and single fraction equivalent dose: useful tools in understanding potency of ablative radiotherapy. Int J Radiat Oncol Biol Phys 70:847-852. https://doi.org/10.1016/j.ijrobp. 2007.10.059

17. Hopewell JW, Millar WT, Lindquist C et al (2013) Application of the concept of biologically effective dose (BED) to patients with vestibular schwannomas treated by radiosurgery. J radiosurgery SBRT 2:257-271

18. Song CW, Cho LC, Yuan J, Dusenbery KE, Griffin RJ, Levitt SH (2013) Radiobiology of stereotactic body radiation therapy/ stereotactic radiosurgery and the linear-quadratic model. Int $\mathbf{J}$ Radiat Oncol Biol Phys 87:18-19. https://doi.org/10.1016/j.jirobp. 2013.03.013

Publisher's note Springer Nature remains neutral with regard to jurisdictional claims in published maps and institutional affiliations. 